\title{
ON POLYNOMIALS CHARACTERIZED BY A CERTAIN MEAN VALUE PROPERTY
}

LEOPOLD FLATTO

Let $\bigvee$ denote the vector space of continuous real valued functions $f(x)$ satisfying the mean value property

$$
f(x)=\frac{1}{N} \sum_{i=1}^{N} f\left(x+t y_{i}\right)
$$

for $x \in R, 0<t<\epsilon_{x}$ ( $R$ denotes an $n$-dimensional region; $x$ and $y_{i}$ are abbreviations for $\left.\left(x_{1}, \cdots, x_{n}\right),\left(y_{i 1}, \cdots, y_{i n}\right)\right)$. We assume that the $y_{i}$ 's span $E_{n}$ so that $1 \leqq n \leqq N$. We furthermore assume, without loss of generality, that $y_{1}, \cdots, y_{n}$ are linearly independent.

Friedman and Littman [5] have recently shown that $V$ consists of polynomials of degrees $\leqq N(N-1) / 2$. This bound is actually attained when the $y_{i}$ 's form the $N$ vertices of an $(N-1)$-dimensional regular simplex [see 4, p. 264]. On the other hand it is known that for $n=2, \operatorname{deg} f \leqq N$ [see 4, Theorem 3.2]. The object of this paper is to obtain bounds on $\operatorname{deg} \vee$ and $\operatorname{dim} \vee$, the bounds depending on $N$ and $n(1 \leqq n \leqq N)$. We use the term deg $\vee$, to denote the maximum degree of the polynomials contained in $V$. We also characterize for fixed $N$ and varying $n(1 \leqq n \leqq N)$ those configurations for which $\operatorname{deg} \bigvee$ and $\operatorname{dim} \bigvee$ attain their maximum.

Theorem. We have

$$
\operatorname{deg} \bigvee \leqq \sum_{j=1}^{n}(N-j), \quad \operatorname{dim} \bigvee \leqq \prod_{j=0}^{n-1}(N-j)
$$

so that for fixed $N$ and varying $n(1 \leqq n \leqq N)$

$$
\operatorname{deg} \bigvee \leqq \frac{N(N-1)}{2}, \quad \operatorname{dim} \bigvee \leqq N ! .
$$

The latter bounds are obtained if and only if

$$
n=N \quad \text { or } \quad n=N-1 \quad \text { and } \quad \sum_{i=1}^{N} y_{i}=0 .
$$

REMARK. The bounds in (2) are not best possible. For instance, we have stated above that for $n=2, \operatorname{deg} \bigvee \leqq N$ and this bound is best

Received by the editors September 7, 1965. 
possible. For fixed $n$ and $N$ the problem of determining the maximum values of $\operatorname{deg} V, \operatorname{dim} V$ and the configurations for which these maximum values are attained remains open.

Proof. We employ the following notation.

$$
\begin{gathered}
\frac{\partial}{\partial x}=\left(\frac{\partial}{\partial x_{1}}, \ldots, \frac{\partial}{\partial x_{n}}\right), \quad x \cdot y=x_{1} y_{1}+\cdots+x_{n} y_{n}, \\
P_{k}(x)=\sum_{i=1}^{N}\left(x \cdot y_{i}\right)^{k} \quad(1 \leqq k<\infty) .
\end{gathered}
$$

It is shown in [5] that (1) is equivalent to the infinite system of homogeneous partial differential equations

$$
P_{k}\left(\frac{\partial}{\partial x}\right) f=0 \quad(1 \leqq k<\infty)
$$

and that $\bigvee$, which is thus the solution space of (4), is a finite dimensional space consisting of polynomials. Let $R$ denote the ring of polynomials in $x_{1}, \cdots, x_{n}$ with real coefficients and let $\mathfrak{B}$ denote the ideal generated by the $P_{k}$ 's $(1 \leqq k<\infty) . R, \mathfrak{B}$, and $\bigvee$ are vector spaces over the reals and it is known that $R$ is the direct sum of $\mathfrak{B}$ and $\vee$, i.e. $R=\mathfrak{B} \oplus \vee$ [see 2 , p. 53]. Thus the vector spaces $R / \mathfrak{P}$ and $\bigvee$ are isomorphic $(R / \mathfrak{B} \cong \bigvee)$.

$\operatorname{deg} V$ and $\operatorname{dim} V$ will thus be determined if we know all the polynomials in $\mathfrak{B}$. We introduce the new variables $\xi_{i}=x \cdot y_{i}(1 \leqq i \leqq N)$. Since the $y_{i}$ 's $(1 \leqq i \leqq N)$ are linearly independent we must have $\xi_{n+k}=\sum_{i=1}^{n} a_{k i} \xi_{i}(1 \leqq k \leqq N-n)$ for an appropriate choice of real $a_{k i}$ 's. Let $R^{\prime}$ denote the ring of polynomials in $\xi_{1}, \cdots, \xi_{n}$ with real coefficients and let $\mathfrak{B}^{\prime}$ denote the ideal generated by the $\eta_{k}$ 's where $\eta_{k}$ $=\sum_{i=1}^{n} \xi_{i}^{k}(1 \leqq k \leqq \infty)$. We adopt the following notation:

$$
\xi=\left(\xi_{1}, \cdots, \xi_{n}\right), i=\left(i_{1}, \cdots, i_{n}\right), \quad \xi_{i}^{i}=\xi_{1}^{i_{1}}, \cdots, \xi_{n}^{i_{n}} .
$$

It is known [see 1, p. 41] that every polynomial $Q(\xi)$ can be expressed as

$$
Q(\xi)=\sum^{\prime} R_{i} \xi^{i},
$$

where the summation in $\sum^{\prime}$ extends over those $i$ s for which $0 \leqq i_{j}$ $\leqq N-j(1 \leqq j \leqq n)$ and $R_{i}$ is a polynomial in $\eta_{1}, \cdots, \eta_{N}$. This representation is unique for $n=N$. Let $c_{i}$ denote the constant term in $R_{i}$ and let $S_{i}=R_{i}-c_{i}$. Clearly $S_{i} \in \mathfrak{P}^{\prime}$. It follows from (5) that $Q(\xi)$ $=\sum^{\prime} C_{i} \xi^{i}+\sum^{\prime} S_{i} \xi^{i}$ so that 


$$
Q(\xi) \equiv \sum^{\prime} c_{i} \xi^{i}\left(\bmod \mathfrak{P}^{\prime}\right) .
$$

As there are $\prod_{j=0}^{n-1}(N-j)$ distinct $\xi^{i}$ s, (6) shows that $\operatorname{dim} R^{\prime} / \mathfrak{P}^{\prime}$ $\leqq \prod_{j=0}^{n-1}(N-j)$. Since $\vee \cong R / \mathfrak{B} \cong R^{\prime} / \mathfrak{P}^{\prime}$ we have $\operatorname{dim} \vee$

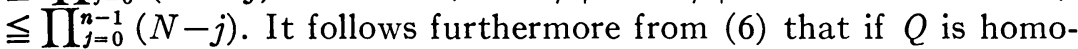
geneous and $\operatorname{deg} Q>\sum_{j=1}^{n}(N-j)$, then $Q \in \mathfrak{P}^{\prime}$. This implies that if $P(x)$ is homogeneous and $\operatorname{deg} P>\sum_{j=1}^{n}(N-j)$ then $P \in \mathfrak{B}$. Thus $\operatorname{deg} \bigvee \leqq \sum_{j=1}^{n}(N-j)$.

If $n \leqq N-2$, then we conclude from (2) that deg $\vee<N(N-1) / 2$, $\operatorname{dim} \bigvee<N$ !. It remains to treat the two cases: (a) $n=N$, (b) $n=N-1$. In case (a) the $\xi^{i}$ 's form a basis for $R^{\prime} / \mathfrak{P}^{\prime}$. For suppose that $\sum^{\prime} c_{i} \xi^{i}$ $\equiv 0\left(\bmod \mathfrak{P}^{\prime}\right)$ for some choice of real $c_{i}$ 's. Then $\sum^{\prime} c_{i} \xi^{i}=\sum_{j=1}^{n} T_{j}(\xi) \eta_{j}$ where the $T_{j}$ 's are polynomials in $\xi_{1}, \cdots, \xi_{n}$. But each $T_{j}$ has a representation (5). I.e. $T_{j}(\xi)=\sum^{\prime} R_{j i}(\eta) \xi^{i}$ where the $R_{j i}$ 's are polynomials in $\eta_{1}, \cdots, \eta_{n}$. Thus $\sum^{\prime} c_{i} \xi^{i}=\sum_{j=1}^{n} \sum^{\prime} R_{j i} \eta_{i} \xi^{i}=\sum^{\prime}\left(\sum_{j=1}^{n} R_{j i} \eta_{j}\right) \xi^{i}$. Since the representation (5) is unique for $n=N$ we have

$$
c_{i}=\sum_{j=1}^{n} R_{j i} \eta_{j}
$$

The left side of (7) is void of $\eta$ 's so that all $R_{j i}$ 's and $c_{i}$ 's equal 0 . Thus $\operatorname{dim} R^{\prime} / \mathfrak{B}^{\prime}=N$ ! and since $\bigvee \cong R / \mathfrak{P} \cong R^{\prime} / \mathfrak{P}^{\prime}, \operatorname{dim} \bigvee=N$ !. Now $\prod_{j=1}^{N-1} \xi_{j}^{N-j}$ has degree $N(N-1) / 2$ and $\in \mathfrak{P}^{\prime}$. This implies that there exists a homogeneous polynomial of degree $N(N-1) / 2 \notin \mathfrak{P}$. Hence $\operatorname{deg} \bigvee=N(N-1) / 2$.

If $n=N-1$ then we distinguish two cases. If $\sum_{i=1}^{N} y_{i} \neq 0$, then it follows from [1, Theorem 2.2] that there exists an orthogonal transformation $x=T x^{\prime}$ such that $g\left(x^{\prime}\right)=f\left(T x^{\prime}\right)$ is independent of $x_{n}^{\prime}$ and satisfies the equation

$$
g\left(x_{p}^{\prime}\right)=\frac{1}{N} \sum_{i=1}^{N} g\left(x_{p}^{\prime}+t y_{p, i}\right),
$$

where $y_{i}=T y_{i}^{\prime}, x_{p}^{\prime}=\left(x_{1}^{\prime}, \cdots, x_{n-1}^{\prime}\right), y_{p i}^{\prime}=\left(y_{i 1}^{\prime}, \cdots, y_{i, n-1}^{\prime}\right)$. Let $\bigvee^{\prime}$ denote the solution space of (8). Clearly deg $\bigvee=\operatorname{deg} \bigvee^{\prime}, \operatorname{dim} \bigvee$ $=\operatorname{dim} \bigvee^{\prime}$. It follows from (2) that $\operatorname{deg} \bigvee=\operatorname{deg} \bigvee^{\prime}<N(N-1) / 2$, $\operatorname{dim} \bigvee=\operatorname{dim} \bigvee^{\prime}<N$ !. If $\sum_{i=1}^{N} y_{i}=0$, then define

$\bar{x}=\left(x_{1}, \cdots, x_{n}, x_{n+1}\right), y_{i}=\left(y_{i 1}, \cdots, y_{i n}, 1\right)(1 \leqq i \leqq N), F(\bar{x})=f(x)$.

We notice that $\sum_{i=1}^{N} \bar{y}_{i} \neq 0$. It therefore follows from [3, Theorem $2.2]$ that $V$ is identical with the solution space $\bar{V}$ of

$$
F(\bar{x})=\frac{1}{N} \sum_{i=1}^{N} F\left(\bar{x}+t \bar{y}_{i}\right) .
$$


Equation (9) is included in Case (a). It follows that deg $V=\operatorname{deg} \nabla$ $=N(N-1) / 2, \operatorname{dim} \bigvee=\operatorname{dim} \bar{V}=N$ !.

\section{REFERENCES}

1. E. Artin, Lectures on Galois theory, Notre Dame Mathematical Lectures, Ann. Arbor, Michigan, 1944.

2. E. Fischer, Über Algebraische Modulsysteme und lineare homogene partiel Differentialgleichungen mit konstaten Koeffizienten, J. Reine Angew. Math. 140 (1911), 48-81.

3. L. Flatto, Functions with a mean value property, J. Math. Mech. 10 (1961), 11-18.

4. - Functions with a mean value property. II, Amer. J. Math. 85 (1963), 248-270.

5. A. Friedman and W. Littman, Functions satisfying the mean value property, Trans. Amer. Math. Soc. 102 (1962), 167-180.

Belfer Graduate School of Sciences, Yeshiva University 